\title{
THE X-RAY NOVA GRO J0422+32 IN DECLINE AND QUIESCENCE
}

\author{
M. R. GARCIA, P. J. CALLANAN, J. E. MCCLINTOCK, \\ P. ZHAO \\ Smithsonian Astrophysical Observatory, Cambridge, MA, USA
}

We have followed the X-ray nova GRO J0422+32, spectroscopically and photometrically, throughout the decline to quiescence.

In the final stages of decay (days 430..880 after the outburst, see Callanan et al. (1995) for the epoch 1995), the equivalent width (EW) of the $\mathrm{H \alpha}$ emission increases monotonically and the $\mathrm{R}$ magnitude decreases monotonically. This suggests that the flux in the $\mathrm{H} \alpha$ line is constant, while the continuum fades. The $\mathrm{H} \alpha$ flux is the product of the $R$ band flux $(F(R)$, arbitrarily scaled to 100 at $R=19 \mathrm{mag}$ ) and the $\mathrm{EW}$, and is shown in the last column of the table below. The $\mathrm{H} \alpha$ flux varies by only $\sim 30 \%$ while the continuum fades by a factor of eight (from $R=19 \mathrm{mag}$ to $R=21.3 \mathrm{mag}$ ). So, to first order, the $\mathrm{H} \alpha$ luminosity is constant in the final stages of decay. While it is generally the case that the emission line EWs in individual dwarf novae also increase during the decay, the exact behavior seen in GRO J0422+32 is not what is seen for dwarf novae (on average). Using the relation between $\mathrm{EW}[\mathrm{H} \beta]$ and $M_{\mathrm{v}}$ given in figure 6 of Patterson (1984), we would expect a factor of $\sim 5$ variation in the $\mathrm{H} \alpha$ flux during days $430 \ldots 880$. The stability of the $\mathrm{H} \alpha$ flux implies that somehow the emission line region is 'disconnected' from the continuum ( $R$-band) emission region.

\begin{tabular}{llrrrc}
\hline Day & Date & $\mathrm{EW}(\AA)$ & $R$ & $F(R)$ & Flux $=\mathrm{EW} \times F(R)$ \\
\hline 430 & 1993 Oct 9/11 & 30 & 19.0 & 100 & 300 \\
580 & 1994 March 10 & 100 & 20.0 & 40 & 400 \\
790 & 1994 Sept 29/30 & 150 & 20.8 & 19 & 285 \\
880 & 1994 Dec 30/31 & 210 & 21.3 & 12 & 250 \\
\hline
\end{tabular}

Many authors have noted that in CVs, the width of the Balmer emission lines is an indicator of the inclination of the system. For any thin disk model this must be the case, as the observed line width will depend on the 
projected velocity (i.e. on $\sin i$ ). If the disks are Keplerian and fill a constant fraction of their Roche lobe, one can easily derive the approximation that the projected velocity will also go like $(M / P)^{1 / 3}$ (see Frank, King \& Raine 1992). In order to investigate this we correlated the Balmer line width measured by Williams (1983) versus the inclinations from Ritter (1994). A clear correlation is seen, although with a wide $\left(\sigma=396 \mathrm{~km} \mathrm{~s}^{-1}\right)$ scatter. If one includes the $\sim(M / P)^{1 / 3}$ factor, the correlation becomes more significant and the scatter drops to $284 \mathrm{~km} \mathrm{~s}^{-1}$. When we add GRO J0422+32 and points for other SXT in quiescence (taken from the literature) to the graph (in larger type) we find that they also seem to obey the same correlation (see figure below).

\section{H-alpha FWHM vs. Orbital Inclination and Mass}

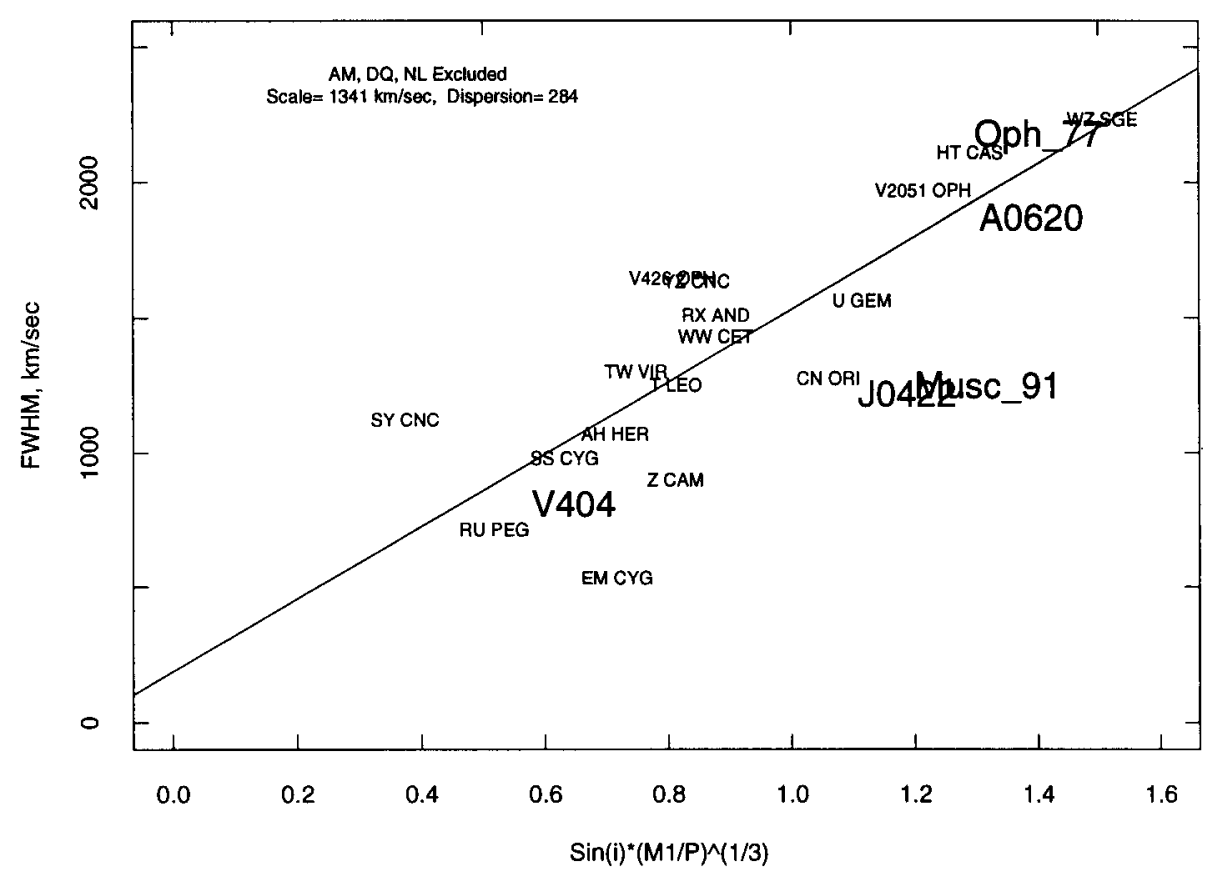

\section{References}

Callanan, P.J., Garcia, M.R., McClintock, J.E., et al., 1995, Ap. J., 441, 786

Frank, J., King, A. R., Raine, D.J., 1992, "Accretion Power In Astrophysics", p54

Patterson, J., 1984, Ap. J. Supp., 54, 443

Ritter, H., 1994, A\&AS, 85, 1179

Williams, G., 1983, Ap. J. Supp., 53, 523 